\title{
Unstable Systems Database: a New Tool for Students, Teachers and Scientists
}

\author{
František Gazdoš, Jiří Marholt and Jaroslav Kolařík \\ Tomas Bata University in Zlin, Faculty of Applied Informatics, nam. T. G. Masaryka 5555, \\ 76001 Zlin, Czech Republic. \\ gazdos@fai.utb.cz
}

\begin{abstract}
The contribution presents a starting project of a site focused on unstable systems. It is a web-based database in the bilingual version (ENG/CZ) which can be used as an information database for models of unstable processes. The site contains mathematical models of such systems including their simulation files together with basic information about stability of dynamical systems. The paper outlines motivation for development of this database, presents its basic structure and discusses several models from the site. Areas of prospective usage are also suggested together with possible directions of further development of this project.
\end{abstract}

\section{Introduction}

Many processes in industrial practice are unstable. These can be e.g. various types of reactors, distillation columns, combustion systems, etc. [1], [2]. There are also lots of systems in the environmental and social fields that are naturally unstable. Besides this, some systems in the military and aviation areas are deliberately designed to be unstable in order to gain better maneuverability and increase speed of command responses. Consequently all these systems are more difficult to control than the stable ones and are of special interest of designers and control engineers. They have to pay extra attention in order to implement safe control systems; if they fail the consequences can be catastrophic [3]. Designers and control engineers have to understand basic limitations that stem from the process instability [4], [5]. As real experiments with unstable systems can be hazardous, modeling and simulation play important roles in designing safe control systems for such processes nowadays. These tools enable safe experiments, analysis of dangerous states of the systems, design of convenient control and much more.

The presented site has been developed to enable students, teachers, scientist and many others interested in unstable processes easy access to mathematical models 
of such systems. All these people can easily use presented models for their own simulation experiments, testing control algorithms, etc. This will broaden awareness about unstable processes and problems they cause. Due to the fact that the database is easily accessible via the Internet [18] it can be used by wide range of users for various purposes, e.g. pedagogical, scientific and others.

The paper is divided into these sections: after a brief introduction into the stability of dynamical systems the contribution continues by examples of unstable processes form the site including their simplified mathematical models together with a brief discussion on their behaviour. Further, a basic structure of the developed site is outlined and explained and the paper concludes suggesting possible further development of the project.

\section{Stability of Dynamical Systems}

Stability is the fundamental property of control systems. Therefore a great deal of effort has been focused towards proper definition, testing and providing for system stability.

\subsection{Defining Stability}

Although all people naturally understand the concept of stability and are able to describe what stable behaviour is and what is not, a proper mathematical definition is not so straightforward. Generally, stability can be formulated as ability to recover from perturbations - short-time disturbances or non-zero initial conditions. One of the definitions says that a system is stable if bounded input into the system produces a bounded output from the system. This is so called BIBO (Bounded Input - Bounded Output) stability, e.g. [6], [7]. Another recognized and more general definition is the Lyapunov stability, e.g. [6], [8], [7], [9]. It states, simply speaking, that a system is stable if its output and all states are bounded and converge asymptotically to zero from sufficiently small initial conditions.

\subsection{Testing Stability}

During the past decades many methods of stability testing have been developed. Usage of a particular method depends on the properties of the system to be tested - e.g. if it is linear or nonlinear, continuous-time or discrete-time, time-variant or time-invariant, etc. The methods can be both numerical and graphical. An interested reader can find details in books focused on systems theory or control engineering, e.g. [6], [7], [9], [10]. 


\subsection{Stabilization}

An unstable system can be stabilized by feedback. There are many sources focused on the control system design for unstable processes, e.g. [1], [11], [12], [13], [2], [14], [15]. Many of these works solve the control system design problem connected also with delayed and non-minimum-phase systems which are also problematic to control.

Besides testing and attainment of stability it is often important to test and ensure certain measure of stability, i.e. relative stability which gives answer to the question how far the system is from instability. For control systems design, so called gain and phase margins are frequently used, for details see e.g. [7], [9], [10].

The so-called robust stability is next important term in control engineering. It is used for the case we want to test/achieve stability not only for one system but for a certain class of systems, typically a nominal system and some its neighborhood, which is useful in the case of uncertain models. An interested reader is referred to books devoted to the robust systems design, e.g. [16], [17].

\section{Examples of Unstable Systems}

As explained in the introduction section unstable processes are common in many areas of our daily lives. Several such systems are briefly described in this section. Mathematical models together with simulation files and original sources are also available online from the developed site [18].

\subsection{Non-ideal CSTR}

This process is represented by a continuous stirred-tank reactor (CSTR) with nonideal mixing. The process can be sketched as illustrated in Figure 1. A simplified mathematical model of the process dynamics can be described by the following nonlinear differential formulas [19], [2]:

$$
\begin{gathered}
\frac{d c(t)}{d t}=\frac{n Q}{m V}\left[c_{f}(t)-c(t)\right]-\frac{k_{1} c(t)}{\left[1+k_{2} c(t)\right]^{2}}, \\
n c(t)+(1-n) c_{f}(t)=c_{e}(t) .
\end{gathered}
$$




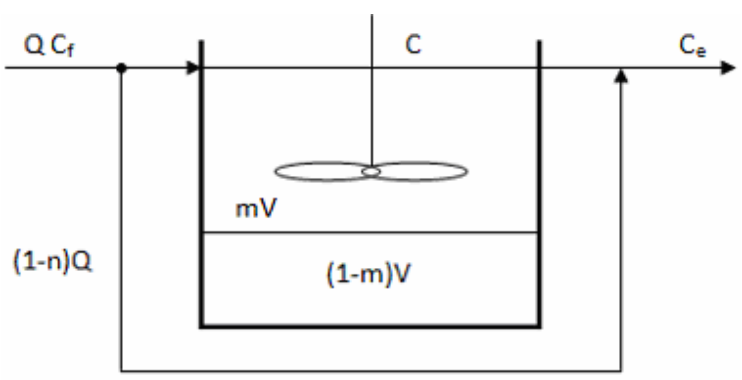

Fig. 1. Non-ideal CSTR

In the picture and equations, $c(t)$ is the concentration of the reactant in the well mixed zone, $c_{e}(t)$ is the concentration in the exit stream (controlled variable) and $c_{f}(t)$ is the feed concentration (manipulated variable). Further, $n$ is the fraction of the reactant feed that enters the zone of perfect mixing and $m$ is the fraction of the reactor total volume $V$ where the reaction occurs. Constants $k_{1}, k_{2}$ describe the reaction rate and $Q$ is the inlet flow rate. Parameters of the model are defined as: $n=m=0.75[-], V=1[1], Q=0.033[1 / \mathrm{s}], k_{1}=10\left[\mathrm{~s}^{-1}\right], k_{2}=10[1 / \mathrm{mol}]$ and a nominal operating point is described as: $c_{f}=6.484[\mathrm{~mol} / \mathrm{l}], c_{e}=1.8[\mathrm{~mol} / \mathrm{l}]$ and $c=$ 0.2387 [mol/l]. Linearization of the nonlinear model (1) around the given nominal operating point gives the transfer function model as:

$$
\frac{\Delta c_{e}(s)}{\Delta c_{f}(s)}=\frac{0.7725 s-0.1727}{3.1 s-1}
$$

A state-space representation of the linearized model in the general form:

$$
\mathbf{x}^{\prime}(t)=\mathbf{A x}(t)+\mathbf{B u}(t) ; \quad \mathbf{y}(t)=\mathbf{C} \mathbf{x}(t)+\mathbf{D u}(t),
$$

where $\mathbf{x}(t)$ defines a vector of state variables, $\mathbf{y}(t)$ a vector of output variables and $\mathbf{u}(t)$ a vector of input variables can be obtained e.g. in this form:

$$
\mathbf{A}=[0.3226], \mathbf{B}=[0.1250], \mathbf{C}=[0.1974], \mathbf{D}=[0.2492]
$$

In the case of the presented reactor the variables $\mathbf{x}(t), \mathbf{y}(t)$ and $\mathbf{u}(t)$ are only scalar and correspond to the reactor variables $c(t), c_{e}(t)$ and $c_{f}(t)$ respectively (consequently the matrices $\mathbf{A}, \mathbf{B}, \mathbf{C}, \mathbf{D}$ are also only scalars). From the control theory point of view, the models (2)-(4) represent a first-order proper system which is unstable (one positive pole, i.e. denominator root, located at $p_{1}=0.3226$ ) 
with non-minimum-phase behaviour (one positive zero, i.e. numerator root, located at $z_{1}=0.2236$ ), and with gain $k=0.1727$ [-]. Such systems which are both unstable and non-minimum-phase are not so easy to control. The step-response of the model recorded in Figure 2 clearly demonstrates instability of the system.

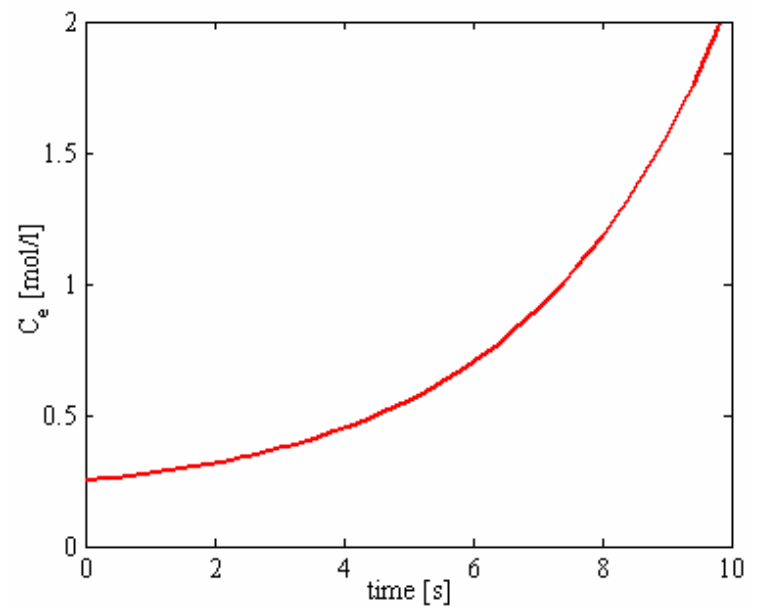

Fig. 2. Non-ideal CSTR step-response

\subsection{Ballistic Missile}

A ballistic missile can represent another unstable system from military industry. Although it is completely different from the previous one, it shares the property of instability and consequently problematic control. When controlling altitude of the ballistic missile, the transfer function relating the altitude $y(t)$ to the thrust chamber deflection $\delta(t)$ has the following form [20], [2]:

$$
\frac{\Delta y(s)}{\Delta \delta(s)}=\frac{7.21(s+0.0526)}{(s+1.6)(s-1.48)(s-0.023)}
$$

Its state-space representation in the general form (3) can be obtained e.g. as:

$$
\mathbf{A}=\left[\begin{array}{ccc}
-0.0970 & 1.1854 & -0.0545 \\
2 & 0 & 0 \\
0 & 0.5 & 0
\end{array}\right], \mathbf{B}=\left[\begin{array}{l}
2 \\
0 \\
0
\end{array}\right], \mathbf{C}=\left[\begin{array}{lll}
0 & 1.8025 & 0.1896
\end{array}\right], \mathbf{D}=[0]
$$


From the control theory point of view the missile represents a strictly proper unstable system of $3^{\text {rd }}$ order. The instability is given by the two poles located in the right half of the complex plane $\left(p_{1}=0.023, p_{2}=1.48\right)$ as illustrated in Figure 3. The system also has relatively fast dynamics with time-constants in seconds.

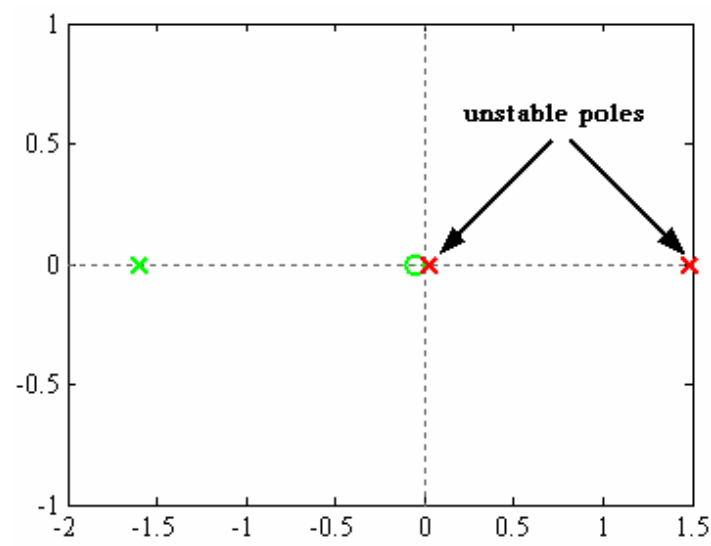

Fig. 3. Pole-zero map of the ballistic missile

\subsection{X-29 Aircraft}

The X-29 illustrated in Figure 4 was an experimental aircraft that tested forwardswept wing, canard control surfaces, and other novel aircraft technologies. It was deliberately designed with static instability to increase its maneuverability and speeds of command response. However, consequently it was impossible to pilot this airplane conventionally by manual flight controls and it required the use of socalled fly-by-wire (computerized) control system. In addition special hardware (sensors, control processors and actuators) had to be used to stabilize the system over all flight regimes and all loading conditions. Considerable effort has been devoted to the design of flight control system for this airplane, e.g. [21], [22], [3].

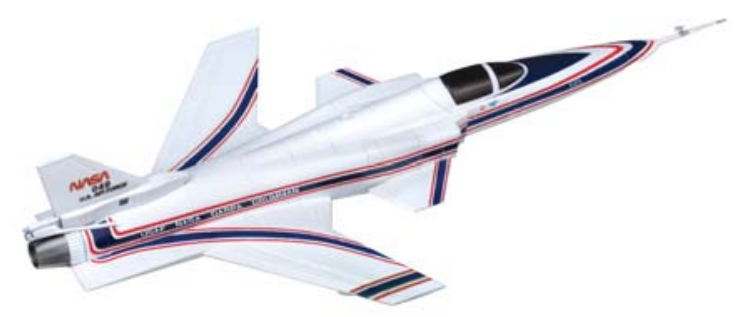

Fig. 4. X-29 aircraft 
The benefits of instability (better maneuverability and faster reaction) were desired in the transonic and supersonic flight regimes, so the airplane was designed to be modestly unstable in those regimes. However, due to a basic aerodynamic phenomenon the X-29's slight instability at supersonic speeds turned into a much more dramatic instability at subsonic speeds. A simplified linearized model at one such flight condition given by a transfer function has the form:

$$
G(s)=\frac{s-26}{s-6}
$$

As can be clearly seen, the airplane's real pole (denominator root) is as large as +6 $\mathrm{rad} / \mathrm{s}$ which makes this system nearly impossible to control manually - it can be compared, simply speaking, to balancing a 1-ft-long stick [3]. Besides the unstable pole the systems has also strong non-minimum-phase behaviour, i.e. inverse response (a zero - numerator root, located at $z_{1}=26$ ). These facts make this system very difficult to control. State-space realization of the model (7) in the general form (3) can be obtained e.g. as:

$$
\mathbf{A}=[6], \mathbf{B}=[4], \mathbf{C}=[-5], \mathbf{D}=[1]
$$

Next figure (Figure 5) shows sensitivity function $S(\omega)$ of a X29 prototype.

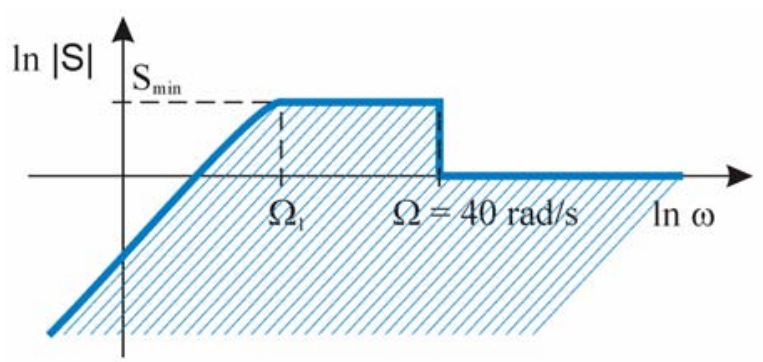

Fig. 5. X-29 sensitivity function

From the plot it can be seen that the system has considerably limited bandwidth (up to $40 \mathrm{rad} / \mathrm{s}$ ) which is given by the used HW components (sensors, control processors, actuators), airplane mechanical structure and aerodynamics conditions. Consequently it narrows possibilities of convenient control system design.

All here discussed models are available online at the presented site http://www.unstable-systems.cz [18] with short description, downloadable model in the MATLAB/Simulink environment and selected references related to modeling, simulation, control system analysis and design of the systems. Next section briefly presents main structure of the developed site devoted to unstable systems and outlines main possibilities it offers for prospective users. 


\section{Site Structure and Choices}

The database of unstable system has been developed as an open, easily extensible system in the bilingual version (English/Czech). It is easily accessible on-line via the Internet at the web-address http://www.unstable-systems.cz. A starting version of the site was implemented within the final work [23] using a free and open source content management system Joomla! of the 2.5 version (available online at http://www.joomla.org and described in detail in e.g. [24]). This makes administration of the site very easy. Basic structure of the web presentation is divided into several bookmarks - HOME, UNSTABLE SYSTEMS, (IN)STABILITY, CONTACT US and ABOUT PROJECT. Apart from this basic structure there are also user-related services such as registration, profile editing and web administration.

The HOME bookmark is the starting point of the site. It introduces a purpose of the site, enables registration, shows latest news and recent posts together with simple statistics and some useful links. Registered users have the following possibilities:

- access to files with simulation models of the systems

- access to latest news via a newsletter

- possibility of articles rating

- possibility to add comments to the systems models

It is also possible to search within the site and change language (ENG/CZ) here.

The UNSTABLE SYSTEMS bookmark is the main part of the site which contains mathematical models of unstable processes. So far (March 2013) it contains following models:

- ballistic missile

- fluidized bed reactor

- inverted pendulum

- magnetic levitation system

- non-ideal continuous stirred-tank reactor

- X-29 aircraft

Every model has the following information:

- brief description of the system

- scheme of the process or picture of the system

- simplified mathematical model

- definition of used variables and parameters

- downloadable model in e.g. MATLAB/Simulink environment

- sources of further information 
It is also possible to generate a printable version of the models or send a link of a model by e-mail. Registered users can download the simulation models, add comments and rate them. Besides this it is also possible to search within the models and sort them according to various criteria.

The (IN)STABILITY bookmark explains basics about stability of systems general understanding of the term, several definitions, such as BIBO (Bounded Input - Bounded Output) and Lyapunov stability. It also offers further reading on this subject.

Next bookmark CONTACT US enables to send questions, suggestions and remarks, etc. on the site and models to the authors. The final bookmark ABOUT PROJECT briefly introduces basic information about this project including main authors, brief description and terms of use.

\section{Conclusions}

Modeling and simulation tools play important roles in our lives nowadays. In the case of unstable systems analysis and control design, they role is crucial. Experiments with such systems without the proper knowledge about possible consequences can be very hazardous [3]. The goal of this contribution was to present a starting project of the web-based database of unstable systems. The site can help students, teachers, designers, scientists and many others to understand basic properties of unstable systems. This is done via available models of the systems/processes and suggested further readings. The developed web-site is an open, constantly developing system which is still "under construction". Therefore experiences of users, their suggestions, remarks and comments are welcome.

Further development of the site will be focused on the extension of the given information concerning the systems stability, description of the models and suggested further readings. The number of available unstable systems models will of course grow as well as the number of simulation files (not necessarily limited to the MATLAB/Simulink environment). Another attractive extension can be seen in the possibility to implement simulation directly into the site, e.g. using the popular Easy Java Simulations (EJS) open-source software tool. At the moment only the site administrator can add articles and models into the system. The possibility to do this by registered users is also being considered, which would certainly help to develop further the database. Besides simulation models of the unstable systems, also simulation files related to control system design for such processes can be added gradually. 


\section{References}

1. Chidambaram, M.: Control of unstable systems: a review. Journal of energy, heat and mass transfer 19, 49-57 (1997)

2. Padma Sree, R., Chidambaram, M.: Control of unstable systems. Alpha science Int. Ltd., Oxford (2006)

3. Stein, G.: Respect the unstable. IEEE Control system magazine 23, 12-25 (2003)

4. Middleton, R.H.: Trade-offs in linear control system design. Automatica 27, 281-292 (1991)

5. Skogestad, S., Havre, K., Larsson, T.: Control limitations for unstable plants. In: Proceedings of the $15^{\text {th }}$ Triennial World Congress, pp. 485-490 (2002)

6. Willems, J.L.: Stability Theory of Dynamical Systems. Wiley, New York (1970)

7. Skogestad, S., Postlethwaite, I.: Multivariable Feedback Control: Analysis and Design. Wiley, Chichester (2005)

8. Parks P.C.: A.M. Lyapunov's stability theory - 100 years on. IMA Journal of Mathematical Control \& Information 4(9), 275-303 (1992)

9. Åström, K.J., Murray, R.M.: Feedback Systems: An Introduction for Scientist and Engineers. Princeton University Press (2008)

10. Doyle, J.C., Francis, B.A., Tannenbaum, A.R.: Feedback Control Theory. Dover Publications (2009)

11.Park, J.H., Sung, S.W., Lee, I.B.: An enhanced PID control strategy for unstable processes. Automatica 34, 751-756 (1998)

12. Marchetti, G., Scali, C., Lewin, D.R.: Identification and control of open-loop unstable processes by relay methods. Automatica 37, 2049-2055 (2001)

13. Lozano, R., Castillo, P., Garcia, P., Dzul, A.: Robust prediction-based control for unstable delay systems: application to the yaw control of a mini-helicopter. Automatica 40, 603-612 (2004)

14. García, P., Albertos, P., Hägglund, T.: Control of unstable non-minimum-phase delayed systems. Journal of Process Control 16, 1099-1111 (2006)

15. Dostál, P., Gazdoš, F., Bobál, V.: Design of controllers for time delay systems - part II: integrating and unstable systems. Journal of electrical engineering 59, 3-8 (2008)

16. Barmish, B.R.: New Tools for Robustness of Linear Systems. Macmillan (1994)

17. Bhattacharyya, S.P., Chapellat, H., Keel, L.H.: Robust Control - The Parametric Approach. Prentice-Hall (1995)

18. Gazdoš, F., Kolařík, J.: Database of unstable systems (2012), http://www.unstable-systems.cz

19. Liou, C.T., Chien, Y.S.: The effect of nonideal mixing on input multiplicities in a CSTR. Chem. Eng. Sci.46, 2113-2116 (1991)

20. Blakelock, J.H.: Automatic Control of Aircraft and Missiles. John Wiley, New York (1991)

21. Rogers, W.L., Collins, D.J.: X-29 $\mathrm{H}_{\infty}$ controller synthesis. J. Guidance control and dynamics 4(15) 962-967 (1992)

22. Clarke, R., Burken, J.J., Bosworth, J.T., Bauer, J.E.: X-29 flight control system - lessons learned. Int. J. Control 1(59), 199-219 (1994)

23. Kolařík, J.: Web-based Database of Unstable Systems. Bachelor's thesis. Tomas Bata University in Zlín, Faculty of Applied Informatics, CZ (2012)

24. Marriott, J., Waring, E.: The official Joomla! Book. Addison-Wesley Professional (2013) 